\title{
The SKA and the Unknown Unknowns
}

\author{
Peter Wilkinson*1 \\ ${ }^{1}$ Jodrell Bank Centre for Astrophysics, School of Physics and Astronomy, The University of \\ Manchester, Manchester, UK;
}

E-mail: peter.wilkinson@manchester.ac.uk

\begin{abstract}
As new scientists and engineers join the SKA project and as the pressures come on to maintain costs within a chosen envelope it is worth restating and updating the rationale for the "Exploration of the Unknown" (EoU). Maintaining an EoU philosophy will prove a vital ingredient for realizing the SKA's discovery potential. Since people make the discoveries enabled by technology a further axis in capability parameter space, the "human bandwidth" is emphasised. Using the morphological approach pioneered by Zwicky, a currently unexploited region of observational parameter space can be identified viz: time variable spectral patterns on all spectral and angular scales - one interesting example would be "spectral transients". We should be prepared to build up to $10 \%$ less collecting area for a given overall budget in order to enhance the ways in which SKA1 can be flexibly utilized.
\end{abstract}

Advancing Astrophysics with the Square Kilometre Array

June 8-13, 2014

Giardini Naxos, Sicily, Italy

\footnotetext{
* Speaker.
} 


\section{Introduction}

The 2014 SKA Science Case has become impressively wide-ranging. Perhaps the most significant change in perception over recent years has been the increased importance afforded to transient science, which can be viewed as an emerging response to the "6th KSP" the Exploration of the Unknown (EoU). Nonetheless the main science imperatives for SKA1 can be traced back to the 2004 Science Case (Carilli \& Rawlings 2004) - and quite often back to the arguments for the "Hydrogen Array" (Wilkinson 1991). The rationale for the SKA has firm foundations.

Despite the growth in breadth and depth of the science case over the past ten years we can still be certain that the SKA's place in astronomical history will not all be found within its pages. As the authors of EoU (Wilkinson et al. 2004) in the 2004 Science Case put it "If history is any example, the excitement of the SKA will not be in the old questions which are answered, but the new questions which will be raised by the new types of observations it alone will permit. The SKA is a tool for as-yet-unborn users and there is an onus on its designers to allow for the exploration of the unknown." More recently Kulkarni (2012) has noted: "discovery is important in astronomy because we are not sufficiently imaginative to construct the Universe and its constituents from first principles..."

The question is how to respond. In 2004 when considering the potential Key Science Programmes the SKA Level Zero Science Committee stated: “... the topic of 'serendipity' does not meet the [level-0] definitions... The recommendation... is therefore that serendipitous discoveries and the expansion of phase space not be included as level-0 science but that serendipity be explicitly included in the science case as an additional motivation for building the SKA." As Carilli put it in his science retrospective to AASKA14 "unknowns were a hard sell in 2004 since physics had torqued astronomy towards experiments but the pendulum is swinging... and general facilities may be back in vogue".

Recent SKA planning documents show that indeed the arc of history is bending in the right direction. In the SKA Concept of Operations Document (Bock et al. 2013) for example: "the primary success metric for the SKA Observatory will be the significance of its role in making fundamental scientific discoveries" and "recognizing the long history of discovery at radio wavelengths... the telescope will be designed in a manner to affordably allow flexibility and evolution of its capabilities to probe new parameter space (e.g., time variable phenomena that current telescopes are not well-equipped to detect)". While in the SKA Level 0 Science Requirements Document (Braun 2014) three of the general scientific requirements of SKA1 are given as:

- to provide the capability to carry out small-scale, experimental observations that utilise the outputs of tied-array beams from one or more sub-arrays.

- to be capable of rapid reconfiguration of their observing mode in response to internal or externally generated, pre-defined triggers.

- to enable commensal observations. It is apparent that many scientific applications can make effective use of the same basic observing campaign to multiply the scientific productivity of the facilities by a large factor. 
Explicit realizations of these philosophies are captured in the specifications of the SKA1 Level 1 System Requirements (Cornwell et al. 2014). This is all greatly to be welcomed. But as pressures come on to keep costs within a chosen ceiling good intentions may fall victim to bean counting.

\section{Discovery in Radio Astronomy - Lessons from History}

In 2007 I assembled a personal list of important discoveries made with classical radio astronomy instruments working from deca to centimetre wavelengths - as is appropriate for SKA (Wilkinson 2007). Space prevents me from presenting a complete updated list here, instead additions to the 2007 list (with thanks to colleagues) are given in Table 1. What are the lessons to be learned?

1. In this region of the spectrum the large telescopes of their day have been at the forefront of discovery. This can be ascribed to the inherently low signal-to-noise ratio of observations indeed it is only with a radio telescope the size of the SKA that one can expect to detect the radio emission from most objects detected in other wavebands.

2. The rate of discovery has slowed in the last ten years (see Table 2) despite the rapid improvement in digital technology which has been well harnessed by the pulsar community. This was the period when LOFAR was being constructed and the VLA was being upgraded to the JVLA so we can hope to recover from this discovery "recession" over the course of this decade. But to me Table 2 is a prima facie argument for the SKA.

3. The designers of did not anticipate what they would be "known for" - a failing shared with HST as pointed out by Norris et al. (2015).

4. In many cases, the people involved had access to lots of telescope time and were not testing any particular theoretical model (not evidenced in the Table).

\section{Discovery with SKA}

\subsection{Parameter Space}

The SKA's place in astronomical history will not all be anticipated within the pages of the 2014 Science Case - so what do we do about it? We start by recognizing that discovery can be planned. As Harwit (1984) noted technology innovation allows the exploration of new areas of capability parameter space whose axes are, broadly:

- sensitivity;

- spatial coverage and or resolution;

- temporal coverage and or resolution;

- spectral coverage and or resolution;

- availability of past observations. 


\begin{tabular}{|c|c|c|c|}
\hline Discovery & Instrument & Design Purpose & $\begin{array}{l}\text { Forseen by } \\
\text { Designers? }\end{array}$ \\
\hline $\begin{array}{l}\text { Interplanetary Scintillation } \\
(1955)\end{array}$ & $\begin{array}{l}\text { Special purpose inter- } \\
\text { ferometer }\end{array}$ & Solar radio astronomy & Partly \\
\hline $\begin{array}{l}\text { Ordered Magnetic Fields in } \\
\text { External Galaxies (1970s) }\end{array}$ & $\begin{array}{l}\text { Westerbork SRT and } \\
\text { Effelsberg }\end{array}$ & $\begin{array}{l}\text { High resolution imaging } \\
\text { General purposes }\end{array}$ & No \\
\hline $\begin{array}{l}\text { Laing-Garrington effect } \\
\text { (1988) }\end{array}$ & $\begin{array}{l}\text { Cambridge } 5 \mathrm{~km} \text { and } \\
\text { Very Large array }\end{array}$ & High resolution imaging & No \\
\hline $\begin{array}{l}\text { IDV is interstellar scintilla- } \\
\text { tion (1992) }\end{array}$ & $\begin{array}{l}\text { Very Large Array } \\
\text { Westerbork SRT }\end{array}$ & High resolution imaging & No \\
\hline Spinning Dust (1996/97) & $\begin{array}{l}\text { COBE and OVRO } \\
40 \mathrm{~m}\end{array}$ & CMB and general purpose & No \\
\hline $\begin{array}{l}\text { Geodetic (GR) Precession in } \\
\text { a binary pulsar (1998) }\end{array}$ & Effelsberg & General purpose & No \\
\hline Winds on Titan (2004) & Global VLBI & Mapping compact sources & No \\
\hline $\begin{array}{l}\text { RRATs-intermittent pulsars } \\
\text { (2006) }\end{array}$ & $\begin{array}{l}\text { Jodrell Bank Lovell } \\
\text { Telescope }\end{array}$ & General Purpose & No \\
\hline Fast Radio Bursts (2006) & Parkes & General purposes & No \\
\hline $\begin{array}{l}\text { Neutron stars above } 2 \text { Msun } \\
(2010)\end{array}$ & GBT & General purposes & No \\
\hline
\end{tabular}

Table 1: Discoveries in deca-centimetric radio astronomy - an update to the list in Wilkinson (2007)

\begin{tabular}{|l|c|}
\hline Decade & Number of discoveries \\
\hline $1930-9$ & 1 \\
$1940-9$ & 2 \\
$1950-9$ & 4 \\
$1960-9$ & 8 \\
$1970-9$ & 6 \\
$1980-9$ & 5 \\
$1990-9$ & 8 \\
$2000-9$ & 4 \\
\hline
\end{tabular}

Table 2: Rate of discovery in the deca-centimetric radio band

Note that the last one is already an addition to Harwit's original thinking. The ready access to archive data has become a major factor in many modern scientific projects. A recent highlight in radio astronomy was the discovery of Fast Radio Bursts (FRBs) in the Parkes pulsar archive some years after the data were taken (Lorimer et al. 2007). Other pulsar discoveries are mentioned below.

In terms of these technologically-driven axes the future looks bright. Even SKA1 will offer a huge boost in sensitivity coupled with a wide field of view for survey throughput plus the ability to sample with high resolutions in the time, frequency and spatial domains. Flexible archiving is also seen as a priority. Thus in terms of technical capability we should expect SKA1 to be able to find 
unexpected phenomena- but only if "the system" allows its users the maximum chance to do so. To encapsulate this idea let us identify a new, more abstract, axis in capability parameter space

- human bandwidth.

Technological innovation alone will not be enough to unlock SKA's full discovery potential. There should be scope for many as many people as possible to gain access to SKA data and for the expression of curiosity in its use.

\subsection{Types of discovery}

The International SKA SWG (Cordes et al. 2006) put it succinctly: "One might think that all of the combinations of key variables have been probed with existing telescopes. This is far from being the case because the so-defined parameter space has been investigated only in very compartmentalized sub-volumes." They went on to extend the rather general arguments adduced by Wilkinson et al. (2004) and introduced a wide range of potential areas for discovery:

1. New objects in known classes.

2. Targeted known phenomena e.g.

- the EoR;

- magnetic fields in structures covering a range of physical scales

- coherent emission from extrasolar planets

3. New Classes of Sources and Phenomena Based on Known Physics, Biology e.g.

- transient sources of many kinds

- new structures e.g in the Cosmic Web

4. The totally unexpected

For the "rare events" under heading 1) the path forward is simple - find more objects. The amount of "surprise" in a data set rises only as the logarithm of the number of independent data elements within it. In the context of astronomical discovery this "logarithmic surprise" idea was first used by Disney (1998). Wilkinson (2007) particularized it for pulsar-related discoveries and the pulsar story was extended by Kramer (2014). For the targeted known phenomena under heading 2) the way forward is basically via the KSPs and PI science - after all these are the "known unknowns". However when we come to headings 3) and 4) new classes of sources and "allowed" phenomena and the complete surprises we need to look in regions of parameter space not previously explored. As Cordes et al. (2006) recognized, the problem arises in defining these areas and so we now turn to this issue.

\subsection{Are there unexplored regions of parameter space?}

As outlined by Wilkinson (2007) a purely phenomenological approach can offers a complement to the thinking described by Cordes et al. (2006) and by Cordes (2007). This is the basis of Zwicky's "morphological" methodology which he developed to a high art in astronomy and other fields (e.g. Zwicky (1969)). Its basis is that all volume elements in parameter space - as long as 
they are not explicitly prohibited - should be considered. Identifying these elements involves freeing one's mind of prejudice and being willing to contemplate unorthodox comparisons. A start for assessing the reach of SKA1 Level 0 science can be made by constructing a cross-linking matrix with the following broad-brush parameters:

- Observing time: short \& long

- Total intensity: high \& low SNR

- Polarised intensity: high \& low SNR

- Spectral patterns: small scale \& large scale

- Spectral resolution: high \& low

- Spatial patterns: small scale \& large scale

- Spatial resolution: high \& low

- Temporal patterns: short term \& long term

- Temporal resolution: high \& low

This leads to a matrix with 18 rows and columns and 144 independent boxes. This matrix is not shown here for reasons of space but the SKA1 Level 0 Science programmes (Braun 2014) do a good job in filling the boxes. There is, however, a set of boxes involving time variable spectra on all angular and spectral scales which is not filled. One example would be "spectral transients".

\subsection{Operational flexibility}

In order to explore observational parameter space as widely as possible some broad flexibility principles were laid out by Wilkinson et al. (2004). It is encouraging that many of these principles are represented, in one form or another, in the Level 0 Science Specifications and the steadily solidifying technical design. However, the cardinal point of this paper is that people make discoveries not technology. The problem is that no one can forecast which right people are going to be in the right place at the right time. The only response must be to seek ways to allow some people access to lots of telescope time and to allow as many different people as possible to gain some access to the data. The essence of the human bandwidth axis is maximising the "brain multiplexing factor". With this in mind let us look at the range of operational modes identified in SKA documents (Bock et al. 2013; Braun 2014; Cornwell et al. 2014). Reduced to their simplest they are:

- Normal

- PI

- Legacy survey

- Targets of Opportunity

- Custom Experiments

- Commensal Observing

A few comments seem worthwhile. First, the normal and TOO modes will be evolutions of existing practice but there is always scope for adding flexibility. For example when the data are being taken for the Legacy Surveys can one open the "collection window" more than is strictly necessary so 
as to add depth to the archived data set and hence increase the post-observation discovery potential? Second, the most radical of the suggestions made by Wilkinson et al. (2004) was to allow a fraction of observing time for high-risk or unproven new-style observations which may be tentative or based on a hunch and hence hard to justify in the formal sense. Here the availability of independent beams and commensal observing will help without compromising conventional observing programs. Custom experiments must be considered on a case-by-case basis - but don't become prey to the criticism " knows the price of everything and the value of nothing". Finally, a constant theme of the SKA should be to seek constructive ways of establishing parallel access to the data. Wider public access is only briefly mentioned in Bock et al. (2013) but it could well be crucial in EoU. "Citizen Science" is a proven route to maximising the "reach" and the recent run of successes of Einstein@home in finding new pulsars (Allen et al. 2013; Knispel et al. 2013; Pletsch 2013) provides graphic evidence. The SKA was born global - so let it be a globally accessible source of data!

\subsection{Synergy with other telescopes}

The sensitivity of the SKA means that for the first time in the history of astronomy, an object detected in any waveband in the electromagnetic spectrum is also likely to be detected in the radio. The opportunities for data linkages with other with ground- (e.g. LSST; ELT; TMT; GMT) and space-based (e.g. JWST; GAIA; EUCLID) telescopes will be enormously greater than with any previous radio telescope. As well as specific bilateral comparisons SKA scientists should make a major commitment to getting the most out of international Virtual Observatories and hence to maximise professional and public access to multi-wavelength data. There was plenty of evidence from talks at AASKA14 that this message is understood by the SKA community.

\subsection{Conclusions}

\footnotetext{
"The known knowns" - read the literature;

"The known unknowns" - write a proposal;

"The unknown unknowns" - hold on to a vision.
}

The current community which is planning and implementing the first phase of the SKA should maintain faith with the scientists of the future by ensuring that the SKA system is prepared for the EoU. This capability will come from a holistic vision of its:

- Large data collecting power: from raw sensitivity and large field-of-view plus multiple beams some of which can be dedicated to specific tasks.

- Some freedom to take risks: from use of independent beams plus new time allocation paradigms.

- Large human bandwidth: allowed by commensal observing; multiple independent beam capability; an in depth data archive coupled with a coordinated strategy for the use of international Virtual Observatories and the citizens of the world.

Much is this is there in present thinking. But the system is not yet built and the flexibility required for the EoU is likely to impose an additional cost burden and hence is vulnerable. What price is 
reasonable? I propose that we should be prepared to build up to $10 \%$ less collecting area for a given overall budget in order to enhance the ways in which this area can be utilized. The penalty may be up to $20 \%$ longer to carry out some projects but this is a graceful degradation and the upside is opening up "opportunity space". I believe that this is likely to prove a good bargain.

\section{References}

Allen B., Knispel B., Cordes, J. et al., Ap.J., 773, 91 (2013)

Bock, D., Dewdney P., Garrington S., et al "Concept of Operations for the SKA Observatory", Document Number SKA.TEL.SE.OPS-SKO-COO-001-0-A, revision B (2013)

Braun, R., "SKA1 Level 0 Science Requirements" Document Number SKA-TEL.SCI-SKO-SRQ001 Revision 1 (2014)

Cornwell, T., McPherson A. \& Diamond, P., "SKA Phase 1 System (Level 1) Requirements Specification" Document Number SKA-OFF.SE.ARC-SKO-SCI-001-3 Revision (2014)

Carilli, C \& Rawlings, S. eds; "Science with the Square Kilometre Array" New Astronomy Reviews (2004)

Cordes J. et al., SKA Memo 85 (2006)

Cordes, J., SKA Memo 97 (2007)

Disney, M., "The comparative discovery potential of any astronomical instrument - or how to make discoveries", unpublished manuscript, (1998).

Harwit, M., "Cosmic Discovery", pub. MIT Press, Cambridge, (1984)

Lorimer, D., Bailes, M., McLaughlin, M., et al., Science, 318, 777 (2007).

Knispel, B., Eatough., R., Kim, H., et al., ApJ, 774, 93 (2013)

Kramer, M., "Pulsar Science with the SKA and its precursors - an overview": in "Transformational Science with the SKA" Stellenbosch (2014)

Kulkarni, S., arXiv:1202.2738v1 [astro-ph.IM] (2012)

Norris, R., Basu, K., Brown, M. et al., 2015, “The SKA Mid-frequency All-sky Continuum Survey: Discovering the unexpected and transforming radio-astronomy", in "Advancing Astrophysics with the Square Kilometre Array", PoS(AASKA14)086

Pletsch, H., Guillemot, L., Allen, B., et al., Astrophys J. Lett. 779, L11, (2013)

Wilkinson, P., in IAU/URSI Symposium 131, "Radio Interferometry - Theory, Techniques and Applications", ed. T.J. Cornwell, Publ. astr. Soc. Pacific, Conference Series Vol. 19, p. 428-432, (1991).

Wilkinson, P., Kellermann, K., Cordes, J. et al., in "Science with the Square Kilometre Array", eds C. Carilli and S. Rawlings, New Astronomy Reviews, 48, p1551. (2004)

Wilkinson, P., in "From Planets to Dark Energy The Modern RadioUniverse", Proceedings of Science (2007).

Zwicky, F.,"Discovery, Invention, Research - Through the Morphological Approach", pub. Macmillan, Toronto, (1969) 